\title{
Design of a Constant-Temperature Incubator for Paper Strip Contamination Detection
}

\author{
Jing Lv ${ }^{1}$ \\ School of Engineering and Technology \\ China University of Geosciences (Beijing) \\ Beijing100083, China \\ e-mail: honeylvjing@163.com
}

\author{
Xingju Liu ${ }^{1}$ \\ School of Engineering and Technology \\ China University of Geosciences (Beijing) \\ Beijing100083, China \\ e-mail: xjsafeda@163.com
}

\author{
Xiang $\mathrm{Yu}^{2^{*}}$ \\ School of Material Science and Technology \\ China University of Geosciences (Beijing) \\ Beijing100083, China \\ *e-mail: yuxiang690625@aliyun.com
}

\begin{abstract}
Currently, hygienic conditions of public tableware and kitchenware have become a hot social concern. This work aimed at solving existing problems in detecting the presence of bacteria, a constant-temperature incubator for the paper strip method was designed and a related temperature-control system was developed. Firstly, the detection method and working principle of the incubator were elaborated upon. Using a paper strip, Escherichia coli (E. coli) was collected from the tableware to be examined. The paper strip was then placed in the incubator for a constant-temperature culture. Through chromogenic reaction, the hygienic condition of the tableware could be qualitatively and quantitatively assessed by analyzing the image information on the paper strip. Subsequently, with regard to the heater band, a wrapping pattern was designed and a suitable material was chosen. Three types of temperature sensors were compared, and a platinum resistor was finally selected and installed at the proper position. Additionally, an electronic thermostat was chosen. Conclusively, the designed incubator, which integrated the constant-temperature culture and image acquisition system, could detect the temperature accurately, reliably, and conveniently in real time, and could also detect the hygienic condition of public tableware rapidly and effectively.
\end{abstract}

Keywords- paper strip method; constant-temperature condition; incubator; Escherichia coli colony; temperaturecontrol system

\section{INTRODUCTION}

When people go out to eat in a public establishment, the hygienic conditions of the tableware and kitchenware should be given priority in consideration. As more and more events are reported in which public tableware and kitchenware do not meet hygienic standards, people are paying increasing attention to the issue. In some dining places, in order to save expenses on labor and electrical costs, almost no disinfecting treatments are performed on the tableware, which severely threatens the consumers' health. The number of Escherichia coli (E. coli) that is present can be an important monitoring index reflecting the disinfection results of tableware [1]. In terms of the detection of bacterial colonies, the microbiological test strip method exhibits several advantages over the multipletube fermentation technique, such as high sensitivity, short detection period, and ease of operation. During the detection process, E. coli on the tableware are collected and placed in an incubator for a constant-temperature culture. By observing the image information on the test strip and the resulting chromogenic reaction, the hygienic condition of tableware can be detected both quantitatively and qualitatively. However, existing domestic products generally have the following problems: (i) The constanttemperature incubation system and the image acquisition system are separate in an incubator, i.e., the temperature at which the images are observed is not always the optimum temperature for growing culture, leading to inaccurate detection results; and (ii) The temperature control systems have several disadvantages, mainly a low temperaturemeasurement accuracy, uneven temperature distribution, and inconvenience of data reading. In foreign products, the costs are often high and maintenance procedures are not convenient. Consequently, a novel constant-temperature incubator for the paper strip method was designed in this study, which perfectly integrated constant-temperature culture and image acquisition. Moreover, the developed incubator provided accurate detection temperature results and convenient operation in real time, by which the hygienic condition of tableware could be detected rapidly and effectively.

\section{DETECTION METHOD}

During the growth and reproduction process, E. coli breaks down lactose and acid is produced. Subsequently, a chromogenic reaction between the acid and the bromcresol purple indicator on the paper strip takes place, causing a yellow circle to appear around the bacterial colony on the paper strip [2]. Meanwhile, due to the existence of the succinodehydrogenase (SDH) produced during the growth of the E. coli, triphenyltetrazolium chloride (TTC) on the paper strip will be irreversibly reduced to formazan, 
resulting in the production of red pigment. Therefore, red dots appear on the paper strip where the E. coli grows. If the whole paper strip or part of the paper strip turns yellow, or red bacterial plaques (or flaky blushes) appear on the yellow background, E. coli is present according to the National Standard (GB. 4789.3-2010) [3]. Comparatively, if the paper strip remains indigo blue or a few purple bacterial plagues (or blushes) appear on that indigo blue background, E. coli is not present.

\section{A. The paper strip for the rapid detection of E. coli.}

The rapid-detection paper strip, which was prepared under sterile conditions through drying at constant temperatures, consists of two layers [4]. The upper layer is a transparent water-retaining coating, which helps the culture medium hold in moisture. Accordingly, the growing conditions of $\mathrm{E}$ coli can be observed in real time. The lower layer is a sterile chromatography paper, on which a certain amount of lactose, indicators (bromcresol purple and TTC), peptone, and fungistat can be absorbed.

\section{B. Reaction process}

1) During the reproduction process, E. coli breaks down lactose and acid is produced.

Lactose - Glucose + Galactose acid

Glucose — Pyruvic acid — Acetic acid + Formic

\section{2) Formic acid $-\mathrm{H} 2+\mathrm{CO} 2$}

\section{Chromogenic reaction}

1) Through a reaction with the produced acid, the bromcresol purple indicator, which is purple initially, turns yellow;

2) The removed hydrogen experiences a redox reaction with the colorless soluble triphenyl tetrazolium chloride (TTC hydrogen acceptor), and insoluble formazan (a kind of red complex) is produced [5].

\section{WORKING PRINCIPLE OF THE INCUBATOR}

The incubator designed in the present work consisted of a box, a shell (mainly including the front and back doors and the upper cover), a temperature-control system, and an image acquisition system, in which the temperaturecontrol system was composed of the heater band, temperature sensor, and temperature controller, while the image acquisition system was composed of a light-emitting diode (LED) and a camera.

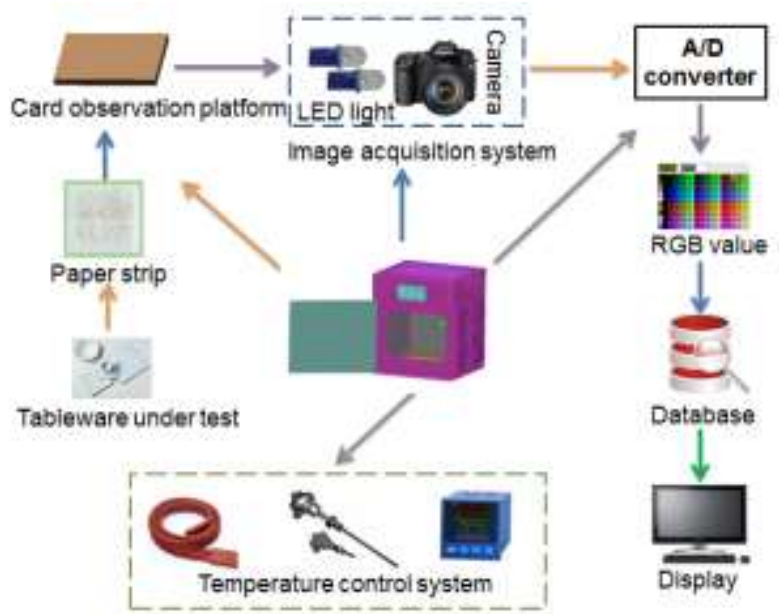

Figure 1. Illustration of working principle of the constant-temperature incubator

Fig .1 illustrates the working principle of the constanttemperature incubator. During testing, the tableware was wiped using the paper strips. These paper strips were then stacked in zip-closure plastic bags. After being sealed, the plastic bags were horizontally placed on the card observation platform in the constant-temperature incubator, and the transparent films were placed face up. It should be noted that no more than 12 pieces of paper strips were stacked. After being cultured for 24 hours at $37{ }^{\circ} \mathrm{C}$, the detection results were observed. Pictures of the paper strips were taken by the image acquisition system (i.e., the camera) in the incubator, and the analog signals of these pictures were then converted into digital signals using an $\mathrm{A} / \mathrm{D}$ converter. Based on the brightness and gray level distributions of the image's pixels, the RGB (Red Green Blue) values of the images were calculated by the computer. By comparing the RGB values to the results in the database, the number of E. coli bacteria was counted, and the hygienic condition of the tested tableware was evaluated.

As stated, the paper strips were placed under a constanttemperature condition at $37{ }^{\circ} \mathrm{C}$ for a 24-hour culture, which was a crucial step in the detection. Under these conditions, the E. coli grew and reproduced well, and then reacted with the culture medium in the strip completely. The detection results were reliable and accurately reflected the contamination degree of the tableware. It was concluded that, when using the paper strip method, the quality of the temperature-control system directly affected the performance of the designed constant-temperature incubator.

\section{DESIGN OF THE TEMPERATURE-CONTROL SYSTEM}

When it came to designing the temperature control system, stability and uniformity of temperature were two significant factors since they concerned the growth of E. coli and thus directly affected the detection results. These two factors were the prerequisites for accurately detecting the hygienic conditions of the tableware.

In the present work, the temperature-control system included three parts, namely, the heater band, temperature sensor, and temperature controller. The design ideas were 
as follow: (1) Determine the control principle of the system; (2) Select the heating device and design the related wrapping pattern; (3) Select and identify the location of the temperature sensor; and (4) Select the temperaturecontroller.

\section{A. Principle for temperature controlling}

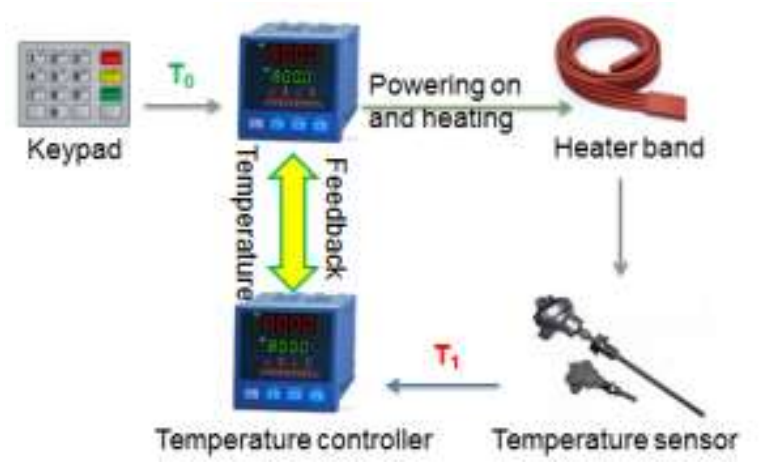

Figure 2. Illustration of control principle of the incubator

Fig .2 illustrates the temperature-control principle of the proposed constant-temperature incubator. After being powered on, the incubator must be initialized firstly in order to judge whether the system runs normally. If faults occur, the incubator should be initialized again. When the system runs normally, the required temperature $T_{0}$ can be set by tuning the temperature-adjustment key in the incubator. After receiving the command, the temperature controller sends heating signals to activate the heater band. Accordingly, the heater band wrapped around the tank starts to heat up, and simultaneously, the temperature sensor installed at the back wall of the box starts to work. The temperature sensor is used for detecting the temperature $\left(\mathrm{T}_{1}\right)$ in the incubator and sending dynamic temperatures to the temperature controller. When the temperature is equivalent to or greater than the set value $\left(\mathrm{T}_{0}\right)$ after receiving feedback from the temperature sensor, the temperature controller will be powered off automatically and the heater band will stop heating. When the temperature in the incubator is lower than the set value $\left(\mathrm{T}_{0}\right)$, the temperature sensor will send a signal to the temperature controller, and the heater band will begin to heat up. These processes are repeated continuously. In order to observe the temperature in real time, a LCD (liquid crystal display) is installed outside the box.

\section{B. Design of the wrapping pattern of the heater band and selection of material}

According to the design requirements of the temperature-control system, favorable temperature uniformity and a rapid heating rate is required [6]. The wrapping pattern directly affects the uniformity of temperature distribution. Fig .3 shows the wrapping pattern of the heater band, and it can be observed that the heater band is uniformly wrapped around the liner clockwise. When receiving the command from the temperature controller, the heater band begins to heat up, and the tank receives the transmitted energy rapidly, so as to ensure that the temperature distribution inside the box is uniform. The heating rate of the temperature-control system depends on the material of the heater band [7]. In the present work, insulated multilayer alkali-free glass fiber was found to be suitable. This material is soft, and can be directly and conveniently wrapped around the tank of the box. Additionally, it is safe and reliable, with adequate thermal endurance and insulating performance and a rapid heattransfer rate.

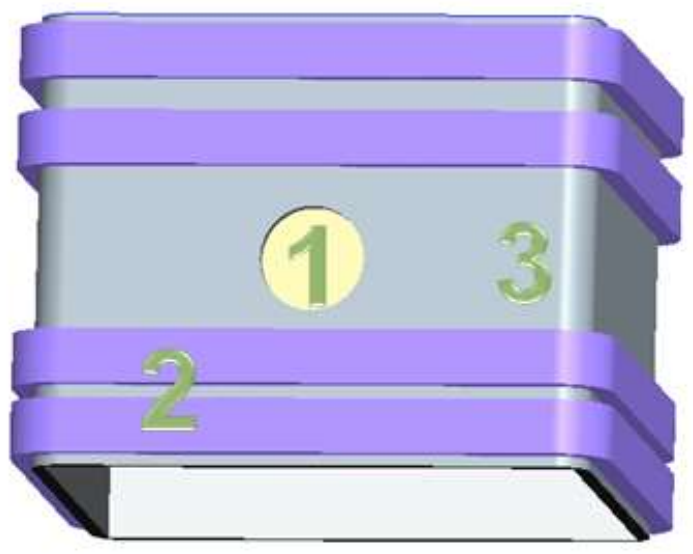

Figure 3. Illustration of wrapping pattern of heater band: (1) The hole for the camera ; (2) Heater band ; (3) Liner

\section{Selection of temperature sensor}

While the E. coli is in the incubator, the detection accuracy and temperature stability should be ensured which are generally determined by the temperature sensor [8]. Thermocouples, thermistors, and platinum resistors are frequently adopted as temperature sensors [9]. Owing to their simple structures, broad application ranges, and wide temperature-measurement ranges, thermocouples can be applied in a number of atmospheric environments. However, they have poor detection precision. Thermistors exhibit small volume, rapid response to temperature variation, and high sensitivity. Unfortunately, their disadvantages are quite obvious. Thermistors have poor linearity, and will be self-heated when the current is large. In terms of temperature-measurement ranges, thermistors are inferior to thermocouples. In terms of stability, thermistors are inferior to platinum resistors. As a type of thermistors made up of platinum, platinum resistors exhibit high accuracy, favorable stability, and excellent linearity superior to thermocouples. All these excellent characteristics make platinum resistors quite applicable in cases where high accuracy is required. With stability and accuracy taken into account, a platinum resistor was an ideal choice in the present design because of its stable physical and chemical properties.

\section{Location of temperature sensor}

The location of the temperature sensor directly affects the accuracy of temperature information acquisition [10]. As mentioned above, the heater band was wrapped around the liner. When the temperature controller sends a command, the heat is transferred from the outside of the liner to the inside, i.e., the central position inside the liner is heated the most slowly. As shown in Fig .4, the temperature at the central position on the back wall of the liner is least stable. If the temperature at this position is stable, the temperature in the whole box is uniform, and 
the temperature as displayed on the LCD outside the box is also stable. Only if the temperature inside the incubator is stable can the number of E. Coli be counted accurately. Accordingly, a platinum resistor was installed at the central position on the back wall of the liner.

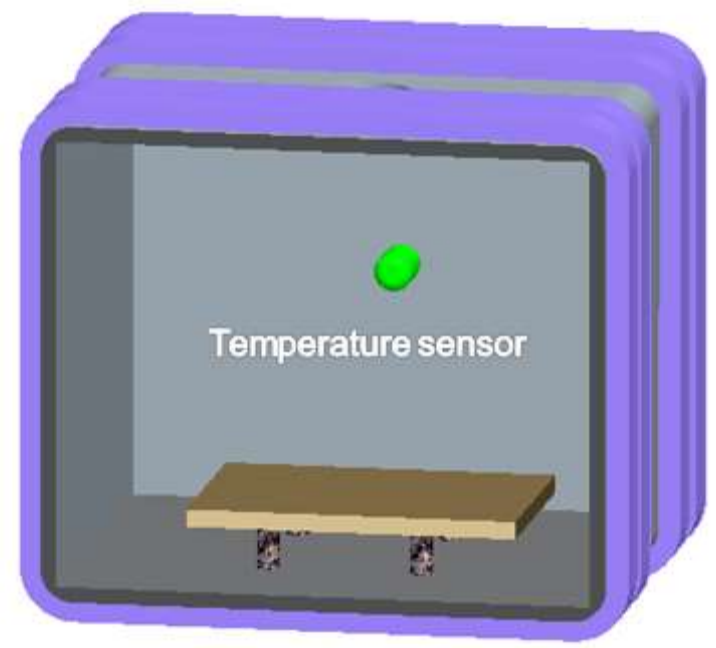

Figure 4. Installation location of the temperature sensor

\section{E. Selection of the temperature controller}

Temperature controllers can be classified as two types, mechanical temperature controllers and electronic temperature controllers [11]. In a mechanical temperature controller, a bimetallic strip or metal bellows is used to adjust the temperature variation according to the expansion and contraction principle. In an electronic temperature controller, the temperature is detected by the temperature sensor, and the operation of the heater is controlled by the connected relay. An electronic temperature controller has a user-friendly operations interface, and can be operated visually. In the present work, an electronic temperature controller was selected and installed on the control panel outside the incubator [12]. Accordingly, the detected temperature value was displayed digitally for the convenience of observation.

\section{CONCLUSIONS}

Focused on the existing problems of detecting bacterial colonies on tableware and kitchenware, a novel constanttemperature incubator for the paper strip method was designed, and the associated temperature-control system was developed. Firstly, the detection method and working principle of the incubator were described, and the temperature-control system was designed [13]. After determining the temperature-control principle, the heater band made up of the insulating multi-layer alkali-free material was selected and uniformly wrapped around the liner in a clockwise direction. Three types of temperature sensors were compared. With stability and accuracy taken into consideration, a platinum resistor was chosen and installed at the central position of the back wall of the liner. Additionally, an electronic temperature controller was selected. Accordingly, the constant-temperature incubation and image acquisition system were integrated in the developed incubator. After the equipment was assembled and testing was complete, the incubator was able to detect temperature accurately, reliably, and conveniently in real time by which the hygienic conditions of tableware could be detected rapidly and effectively [14].

\section{ACKNOWLEDGEMENT}

This work was supported by the Fundamental Research Funds for the Central Universities.

\section{REFERENCES}

[1] Y. S. Jiang and X. Fei, "Technology Progress for Detection of Coliform in Foods," Culinary Science Journal of Yangzhou University, vol. 3, 2013, pp. 18-22.

[2] Y. F. Guo and F. Luo, "Recent Progress in Detection Methods of E. coli in Food and Water," Animal Husbandry and Feed Science, vol. 31, Sep. 2010, pp. 90-91, doi:10.16003/j.cnki.issn16725190.2010.09.011.

[3] National Standard of the People's Republic of China. GB 4789. 3 2010 Food Microbiological Examination: Enumeration of Coliforms [S]. Issued by Ministry of Health of the People's Republic of China.

[4] E. Wollants, P. Maes, I. Thoelen, F. Vanneste, M. Rahman and M. V. Ranst, "Evaluation of a norovirus sampling method using sodium dodecyl sulfate," J. Virol. Methods, vol. 122(1), Dec. 2004, pp. 45-48, doi:10.1016/j.jviromet.2004.08.001.

[5] N. Wang, M. He and H. C. Shi, "Novel indirect enzyme-linked immunesorbent assay (ELISA) method to detect Total E. coli in water environment," Analytica chemical acta, vol. 590(2), May. 2007, pp. 224-231, doi:10.1016/j.aca.2007.03.041.

[6] W. H. Liang and K. Wang, "Design of Intelligent Temperature and Humidity Control System for Vegetable Greenhouse," Journal of Anhui Agricultural Sciences, vol. 19, 2009, pp. 9138-9139.

[7] Y. G. Peng and W. Wei, "Artificial climate chest temperature and humidity fuzzy control based on neural network compensation,' Chinese Journal of Scientific Instrument, Jul. 2009, pp. 1373-1376.

[8] A. G. Cridge, J. Visweswaraiah, R. Ramesh and E. Sattlegger, "Semi-quantitative colony immunoassay for determining and optimizing protein expression in Saccharomyces cerevisiae and Escherichia coli.," Anal. Biochem, vol. 447, Feb. 2014, pp. 82-89, doi:10.1016/j.ab.2013.10.020.

[9] Y. Feng, "Design of temperature control system based on AT89C52," Journal of Weinan Teachers University, vol. 26(2), 2011, pp. 49-52.

[10] J. Zhang, "Smart temperature sensor DS18B20 and its application," Instrum. Sci. Technol, vol. 4, 2010, pp. 68-70.

[11] C. H. Yu and D. P. Zhang, "An artificial intelligence central airconditioning controller," Advances in Intelligent Systems, vol. 138, 2012, pp. 19-25.

[12] S. Thakore, B. Patel, L. Lam, J. Goldfarb and A. Ahmady, "The effect of incubator type on mouse embryo development conventional box versus top-load bench incubators," Fertil. Steril,

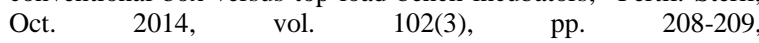
doi.org/10.1016/j.fertnstert.2014.07.704.

[13] D. M. Birch and J. F. Morrison, "An innovative low-profile monolithic constant-temperature anemometer," J. Wind. Eng. Ind. Aerod, vol. 100(1), Jan. 2012, pp. 38-45, doi:10.1016/j.jweia.2011.10.010.

[14] S. K. Chong and M. S. Mohamad, "A hybrid of ant colony optimization and minimization of metabolic adjustment to improve the production of succinic acid in Escherichia coli.," Comput. Biol. Med, vol. 49, Jun. 2014, pp. 74-82, doi:10.1016/j.compbiomed.2014.03.011. 